\title{
New Robust Tracking and Stabilization Methods for Significant Classes of Uncertain Linear and Nonlinear Systems
}

\author{
Laura Celentano \\ Dipartimento di Informatica e Sistemistica \\ Università degli Studi di Napoli Federico II, Napoli, \\ Italy
}

\section{Introduction}

There exist many mechanical, electrical, electro-mechanical, thermic, chemical, biological and medical linear and nonlinear systems, subject to parametric uncertainties and non standard disturbances, which need to be efficiently controlled. Indeed, e.g. consider the numerous manufacturing systems (in particular the robotic and transport systems,...) and the more pressing requirements and control specifications in an ever more dynamic society. Despite numerous scientific papers available in literature (Porter and Power, 1970)-(Sastry, 1999), some of which also very recent (Paarmann, 2001)-(Siciliano and Khatib, 2009), the following practical limitations remain:

1. the considered classes of systems are often with little relevant interest to engineers;

2. the considered signals (references, disturbances,...) are almost always standard (polynomial and/or sinusoidal ones);

3. the controllers are not very robust and they do not allow satisfying more than a single specification;

4. the control signals are often excessive and/or unfeasible because of the chattering. Taking into account that a very important problem is to force a process or a plant to track generic references, provided that sufficiently regular, e.g. the generally continuous piecewise linear signals, easily produced by using digital technologies, new theoretical results are needful for the scientific and engineering community in order to design control systems with non standard references and/or disturbances and/or with ever harder specifications.

In the first part of this chapter, new results are stated and presented; they allow to design a controller of a SISO process, without zeros, with measurable state and with parametric uncertainties, such that the controlled system is of type one and has, for all the possible uncertain parameters, assigned minimum constant gain and maximum time constant or such that the controlled system tracks with a prefixed maximum error a generic reference with limited derivative, also when there is a generic disturbance with limited derivative, has an assigned maximum time constant and guarantees a good quality of the transient.

The proposed design techniques use a feedback control scheme with an integral action (Seraj and Tarokh, 1977), (Freeman and Kokotovic, 1995) and they are based on the choice of a 
suitable set of reference poles, on a proportionality parameter of these poles and on the theory of externally positive systems (Bru and Romero-Vivò, 2009).

The utility and efficiency of the proposed methods are illustrated with an attractive and significant example of position control.

In the second part of the chapter it is considered the uncertain pseudo-quadratic systems of the type $\ddot{y}=F_{1}(y, \dot{y}, p) u+\left[\sum_{i=1}^{m} F_{2 i}(y, \dot{y}, p) \dot{y}_{i}\right] \dot{y}+f(t, y, \dot{y}, p)$, where $t \in R$ is the time, $y \in R^{m}$ is the output, $u \in R^{r}$ is the control input, $p \in \wp \subset R^{\mu}$ is the vector of uncertain parameters, with $\wp$ compact set, $F_{1} \in R^{m \times r}$ is limited and of rank $m, F_{2 i} \in R^{m \times m}$ is limited and $f \in R^{m}$ is limited and models possible disturbances and/or particular nonlinearities of the system.

For this class of systems, including articulated mechanical systems, several theorems are stated which easily allow to determine robust control laws of the PD type, with a possible partial compensation, in order to force $y$ and $\dot{y}$ to go to rectangular neighbourhoods (of the origin) with prefixed areas and with prefixed time constants characterizing the convergence of the error. Clearly these results allow also designing control laws to take and hold a generic articulated system in a generic posture less than prefixed errors also in the presence of parametric uncertainties and limited disturbances.

Moreover the stated theorems can be used to determine simple and robust control laws in order to force the considered class of systems to track a generic preassigned limited in "acceleration" trajectory, with preassigned majorant values of the maximum "position and/or velocity" errors and preassigned increases of the time constants characterizing the convergence of the error.

\section{Part I}

\section{Problem formulation and preliminary results}

Consider the SISO n-order system, linear, time-invariant and with uncertain parameters, described by

$$
\dot{x}=A x+B u, \quad y=C x+d,
$$

where: $x \in R^{n}$ is the state, $u \in R$ is the control signal, $d \in R$ is the disturbance or, more in general, the effect $y_{d}$ of the disturbance $d$ on the output, $y \in R$ is the output, $A^{-} \leq A \leq A^{+}, B^{-} \leq B \leq B^{+}$and $C^{-} \leq C \leq C^{+}$.

Suppose that this process is without zeros, is completely controllable and that the state is measurable.

Moreover, suppose that the disturbance $d$ and the reference $r$ are continuous signals with limited first derivative (see Fig. 1).

A main goal is to design a linear and time invariant controller such that:

1. $\forall A \in\left[A^{-}, A^{+}\right], \forall B \in\left[B^{-}, B^{+}\right], \forall C \in\left[C^{-}, C^{+}\right]$the control system is of type one, with constant gain $K_{v} \geq \hat{K}_{v}$ and maximum time constant $\tau_{\max } \leq \hat{\tau}_{\max }$, where $\hat{K}_{v}$ and $\hat{\tau}_{\max }$ are design specifications, or

2. condition 1. is satisfied and, in addition, in the hypothesis that the initial state of the control system is null and that $r(0)-d(0)=0$, the tracking error $e(t)$ satisfies relation 


$$
|e(t)| \leq \frac{1}{\hat{K}_{v}} \hat{\delta}_{\dot{r}-\dot{d}}, \quad \forall t \geq 0, \quad \forall r(t), d(t): \delta_{\dot{r}-\dot{d}}=\max _{\sigma \in[0, t]}|\dot{r}(\sigma)-\dot{d}(\sigma)| \leq \hat{\delta}_{\dot{r}-\dot{d}},
$$
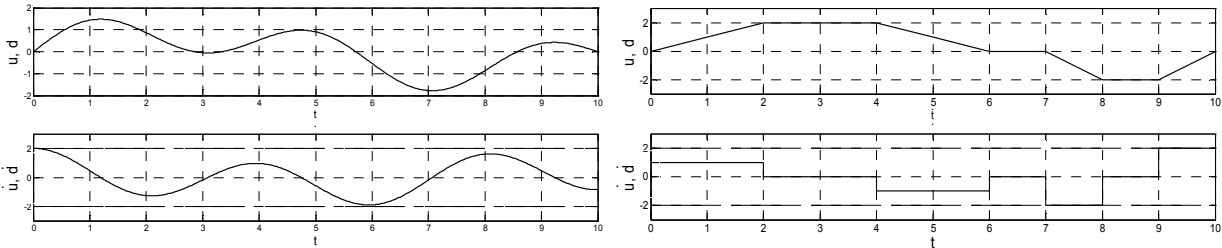

Fig. 1. Possible reference or disturbance signals with limited derivative.

where the maximum variation velocity $\hat{\delta}_{\dot{r}-\dot{d}}$ of $r(t)-d(t)$ is a design specification.

Remark 1. Clearly if the initial state of the control system is not null and/or $r(0)-d(0) \neq 0$ (and/or, more in general, $r(t)-d(t)$ has discontinuities), the error $e(t)$ in (2) must be considered unless of a "free evolution", whose practical duration can be made minus that a preassigned settling time $\hat{t}_{a}$.

Remark 2. If disturbance $d$ does not directly act on the output $y$, said $y_{d}$ its effect on the output, in (2) $\dot{d}$ must be substituted with $\dot{y}_{d}$.

This is one of the main and most realistic problem not suitable solved in the literature of control (Porter et al., 1970)-(Sastry, 1999).

There exist several controllers able to satisfy the 1. and/or 2 specifications. In the following, for brevity, is considered the well-known state feedback control law with an integral (I) control action (Seraj and Tarokh, 1977), (Freeman and Kokotovic, 1995).

By posing

$$
G(s)=C(s I-A)^{-1} B=\frac{b}{s^{n}+a_{1} s^{n-1}+\ldots+a_{n}}, \quad a_{1}^{-} \leq a_{1} \leq a_{1}^{+}, \ldots, a_{n}^{-} \leq a_{n} \leq a_{n}^{+}, b^{-} \leq b \leq b^{+},
$$

in the Laplace domain the considered control scheme is the one of Fig. 2.

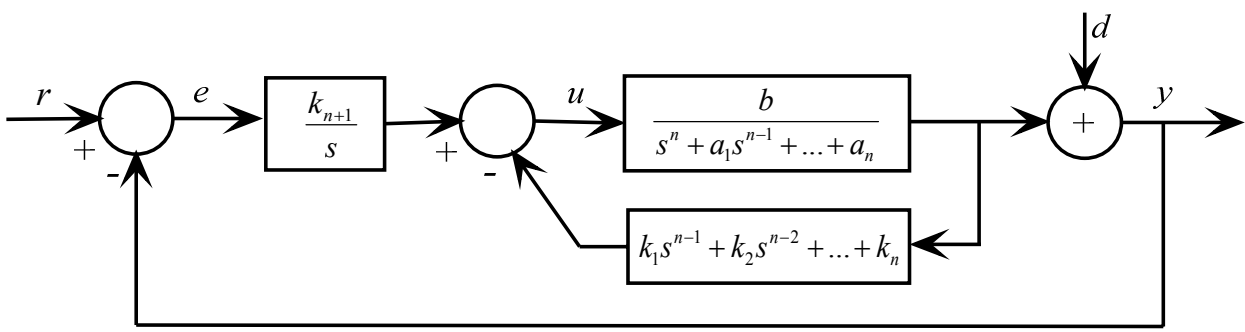

Fig. 2. State feedback control scheme with an I control action.

Remark 3. It is useful to note that often the state-space model of the process (1) is already in the corresponding companion form of the input-output model of the system (3) (think to the case in which this model is obtained experimentally by using e.g. Matlab command invfreqs); on the contrary, it is easy to transform the interval uncertainties of $A, B, C$ into the ones (even if more conservative) of $a_{i}, b$. 
Moreover note that almost always the controller is supplied with an actuator having gain $g_{a}$. In this case it can be posed $b \leftarrow b g_{a}$ and also consider the possible uncertainty of $g_{a}$.

Finally, it is clear that, for the controllability of the process, the parameter $b$ must be always not null. In the following, without loss of generality, it is supposed that $b^{-}>0$.

Remark 4. In the following it will be proved that, by using the control scheme of Fig. 2, if (2) is satisfied then the overshoot of the controlled system is always null.

From the control scheme of Fig. 2 it can be easily derived that

$$
E(s)=s \frac{s^{n}+\left(a_{1}+b k_{1}\right) s^{n-1}+\ldots+\left(a_{n}+b k_{n}\right)}{s^{n+1}+\left(a_{1}+b k_{1}\right) s^{n}+\ldots+\left(a_{n}+b k_{n}\right) s+b k_{n+1}}(R(s)-D(s))=S(s)(R(s)-D(s)) .
$$

If it is posed that

$$
d(s)=s^{n+1}+\left(a_{1}+b k_{1}\right) s^{n}+\ldots+\left(a_{n}+b k_{n}\right) s+b k_{n+1}=s^{n+1}+d_{1} s^{n}+\ldots+d_{n} s+d_{n+1},
$$

from (4) and by noting that the open loop transfer function is

$$
F(s)=\frac{k_{n+1}}{s} \frac{b}{s^{n}+\left(a_{1}+b k_{1}\right) s^{n-1}+\ldots+\left(a_{n}+b k_{n}\right)}=\frac{d_{n+1}}{s\left(s^{n}+d_{1} s^{n-1}+\ldots+d_{n}\right)},
$$

the sensitivity function $S(s)$ of the error and the constant gain $K_{v}$ turn out to be:

$$
S(s)=s \frac{s^{n}+d_{1} s^{n-1}+\ldots+d_{n}}{s^{n+1}+d_{1} s^{n}+\ldots+d_{n} s+d_{n+1}}, \quad K_{v}=\frac{d_{n+1}}{d_{n}}=\frac{b k_{n+1}}{a_{n}+b k_{n}} .
$$

Moreover the sensitivity function $W(s)$ of the output is

$$
W(s)=\frac{d_{n+1}}{s^{n+1}+d_{1} s^{n}+\ldots+d_{n} s+d_{n+1}} .
$$

Definition 1. A symmetric set of $n_{n+1}^{n+1}$ negative real part complex numbers $\bar{P}=\left\{\bar{p}_{1}, \bar{p}_{2}, \ldots, \bar{p}_{n+1}\right\}$ normalized such that $\prod_{i=1}^{n+1}\left(-\bar{p}_{i}\right)=1$ is said to be set of reference poles.

Let be

$$
\bar{d}(s)=s^{n+1}+\bar{d}_{1} s^{n}+\ldots+\bar{d}_{n} s+\bar{d}_{n+1}
$$

the polynomial whose roots are a preassigned set of reference poles $\bar{P}$. By choosing the poles $P$ of the control system equal to $\rho \bar{P}$, with $\rho$ positive, it is

$$
d(s)=s^{n+1}+\rho \bar{d}_{1} s^{n}+\ldots+\rho^{n} \bar{d}_{n} s+\rho^{n+1} \bar{d}_{n+1} .
$$

Moreover, said $s_{p}(t)$ the impulsive response of the system having transfer function

$$
S_{p}(s)=\frac{1}{s} S(s)=\frac{s^{n}+d_{1} s^{n-1}+\ldots+d_{n}}{s^{n+1}+d_{1} s^{n}+\ldots+d_{n} s+d_{n+1}},
$$

from (4) and from the first of (7) it is 


$$
|e(t)| \leq \int_{0}^{t}\left|s_{p}(\tau)\right||\dot{r}(t-\tau)-\dot{d}(t-\tau)| d \tau, \quad \text { where } s_{p}(t)=\mathcal{L}^{-1}\left(S_{p}(s)\right),
$$

from which, if all the poles of $S_{p}(s)$ have negative real part, it is

$$
|e(t)| \leq \frac{1}{H_{v}} \delta_{\dot{r}-\dot{d}}
$$

where

$$
H_{v}=\frac{1}{\int_{0}^{\infty}\left|S_{p}(\tau)\right| d \tau}, \quad \delta_{\dot{r}-\dot{d}}=\max _{\sigma \in[0, t]}|\dot{r}(\sigma)-\dot{d}(\sigma)| .
$$

Remark 5. Note that, while the constant gain $K_{v}$ allows to compute the steady-state tracking error to a ramp reference signal, $H_{v}$, denoted absolute constant gain, allows to obtain $\forall t$ an excess estimate of the tracking error to a generic reference with derivative. On this basis, it is very interesting from a theoretical and practical point of view, to establish the conditions for which $H_{v}=K_{v}$.

In order to establish the condition necessary for the equality of the absolute constant gain $H_{v}$ with the constant gain $K_{v}$ and to provide some methods to choose the poles $\bar{P}$ and $\rho$, the following preliminary results are necessary. They concern the main parameters of the sensitivity function $W(s)$ of the output and the externally positive systems, i.e. the systems with non negative impulse response.

Theorem 1. Let be $\bar{s}, \bar{t}_{s}, \bar{t}_{a}, \bar{\omega}_{s}$ the overshoot, the rise time, the settling time and the upper cutoff angular frequency of

$$
\bar{W}(s)=\frac{\bar{d}_{n+1}}{\bar{d}(s)}=\frac{\bar{d}_{n+1}}{s^{n+1}+\bar{d}_{1} s^{n}+\ldots+\bar{d}_{n} s+\bar{d}_{n+1}}
$$

and

$$
\bar{K}_{v}=\frac{\bar{d}_{n+1}}{\bar{d}_{n}}, \quad \bar{H}_{v}=\frac{1}{\int_{0}^{\infty}\left|\bar{s}_{p}(\tau)\right| d \tau}, \quad \text { where } \quad \bar{s}_{p}(t)=\mathcal{L}^{-1}\left(\frac{s^{n}+\bar{d}_{1} s^{n-1}+\ldots+\bar{d}_{n}}{s^{n+1}+\bar{d}_{1} s^{n}+\ldots+\bar{d}_{n} s+\bar{d}_{n+1}}\right),
$$

then the corresponding values of $s, t_{s}, t_{a}, \omega_{s}, K_{v}, H_{v}$ when $\rho \neq 1$ turn out to be:

$$
s=\bar{s}, \quad t_{s}=\frac{\bar{t}_{s}}{\rho}, \quad t_{a}=\frac{\bar{t}_{a}}{\rho}, \quad \omega_{s}=\rho \bar{\omega}_{s}, \quad K_{v}=\rho \bar{K}_{v}, \quad H_{v}=\rho \bar{H}_{v} .
$$

Proof. By using the change of scale property of the Laplace transform, (8) and (10) it is

$$
\begin{aligned}
w_{-1}\left(\frac{t}{\rho}\right) & =\mathcal{L}^{-1}\left(\rho \frac{\rho^{n+1} \bar{d}_{n+1}}{(\rho s)^{n+1}+\rho \bar{d}_{1}(\rho s)^{n}+\ldots+\rho^{n} \bar{d}_{n}(\rho s)+\rho^{n+1} \bar{d}_{n+1}} \frac{1}{\rho s}\right)= \\
& =\mathcal{L}^{-1}\left(\frac{\bar{d}_{n+1}}{s^{n+1}+\bar{d}_{1} s^{n}+\ldots+\bar{d}_{n} s+\bar{d}_{n+1} s} \frac{1}{s}\right)=\bar{w}_{-1}(t) .
\end{aligned}
$$


By using again the change of scale property of the Laplace transform, by taking into account (10) and (11) it is

$$
\begin{aligned}
s_{p}\left(\frac{t}{\rho}\right)= & \mathcal{L}^{-1}\left(\rho \frac{(\rho s)^{n}+\rho \bar{d}_{1}(\rho s)^{n-1}+\ldots+\rho^{n} \bar{d}_{n}}{(\rho s)^{n+1}+\rho \bar{d}_{1}(\rho s)^{n}+\ldots+\rho^{n} \bar{d}_{n}(\rho s)+\rho^{n+1} \bar{d}_{n+1}}\right)= \\
& =\mathcal{L}^{-1}\left(\frac{s^{n}+\bar{d}_{1} s^{n-1}+\ldots+\bar{d}_{n}}{s^{n+1}+\bar{d}_{1} s^{n}+\ldots+\bar{d}_{n} s+\bar{d}_{n+1}}\right)=\bar{s}_{p}(t)
\end{aligned}
$$

from which

$$
\int_{0}^{\infty}\left|S_{p}(\tau)\right| d \tau=\frac{1}{\rho} \int_{0}^{\infty}\left|S_{p}\left(\frac{t}{\rho}\right)\right| d t=\frac{1}{\rho} \int_{0}^{\infty}\left|\bar{s}_{p}(t)\right| d t .
$$

From the second of (7) and from (10), (14), (18), (20) the proof easily follows.

Theorem 2. Let be $\bar{a}_{i} \in\left[a_{i}^{-}, a_{i}^{+}\right], i=1,2, \ldots, n$, and $\bar{b} \in\left[b^{-}, b^{+}\right]$the nominal values of the parameters of the process and $\hat{P}=\hat{\rho} \bar{P}$ the desired nominal poles. Then the parameters of the controller, designed by using the nominal parameters of the process and the nominal poles, turn out to be:

$$
\hat{k}_{i}=\frac{\hat{\rho}^{i} \bar{d}_{i}-\bar{a}_{i}}{\bar{b}}, i=1,2, \ldots, n, \quad \hat{k}_{n+1}=\frac{\hat{\rho}^{n+1} \bar{d}_{n+1}}{\bar{b}} .
$$

Moreover the polynomial of the effective poles and the constant gain are:

$$
\begin{gathered}
d(s)=\hat{d}(s)+h \hat{n}(s)+\delta(s) \\
K_{v}=\frac{\hat{\rho}^{n+1} \bar{d}_{n+1}}{\frac{a_{n}}{1+h}-\bar{a}_{n}+\hat{\rho}^{n} \bar{d}_{n}}=\frac{\hat{d}_{n+1}}{\frac{a_{n}}{1+h}-\bar{a}_{n}+\hat{d}_{n}},
\end{gathered}
$$

where:

$$
\begin{gathered}
\hat{d}(s)=s^{n+1}+\bar{d}_{1} \hat{\rho} s^{n}+\ldots+\bar{d}_{n} \hat{\rho}^{n} s+\bar{d}_{n+1} \hat{\rho}^{n+1}=s^{n+1}+\hat{d}_{1} s^{n}+\ldots+\hat{d}_{n} s+\hat{d}_{n+1} \\
\hat{n}(s)=\bar{d}_{1} \hat{\rho} s^{n}+\ldots+\bar{d}_{n} \hat{\rho}^{n} s+\bar{d}_{n+1} \hat{\rho}^{n+1}=\hat{d}_{1} s^{n}+\ldots+\hat{d}_{n} s+\hat{d}_{n+1} \\
h=\frac{\Delta b}{\bar{b}}, \quad \delta(s)=\bar{a}_{1}\left(\frac{\Delta a_{1}}{\bar{a}_{1}}-\frac{\Delta b}{\bar{b}}\right) s^{n}+\ldots+\bar{a}_{n}\left(\frac{\Delta a_{n}}{\bar{a}_{n}}-\frac{\Delta b}{\bar{b}}\right) s \\
\Delta b=b-\bar{b}, \quad \Delta a_{1}=a_{1}-\bar{a}_{1}, \ldots, \Delta a_{n}=a_{n}-\bar{a}_{n} .
\end{gathered}
$$

Proof. The proof is obtained by making standard manipulations starting from (5), from the second of (7) and from (10). For brevity it has been omitted.

Theorem 3. The coefficients $d$ of the polynomial

$$
d(s-\hat{\alpha})=s^{n+1}+\left[s^{n} s^{n-1} \ldots s \quad 1\right] d, \quad \hat{\alpha}=1 / \hat{\tau}_{\max },
$$


where $d(s)$ is the polynomial (5) or (22), are given by using the affine transformation

$$
d=\left[\begin{array}{ccccc}
1 & 0 & \cdot & 0 & \hat{\chi}_{1} \\
\left(\begin{array}{l}
n \\
1
\end{array}\right) \hat{\alpha} & 1 & \cdot & 0 & \hat{\chi}_{2} \\
\cdot & \cdot & \cdot & \cdot & \cdot \\
\left(\begin{array}{c}
n \\
n-1
\end{array}\right) \hat{\alpha}^{n-1} & \left(\begin{array}{c}
n-1 \\
n-2
\end{array}\right) \hat{\alpha}^{n-2} & \cdot & 1 & \hat{\chi}_{n} \\
\left(\begin{array}{l}
n \\
n
\end{array}\right) \hat{\alpha}^{n} & \left(\begin{array}{c}
n-1 \\
n-1
\end{array}\right) \hat{\alpha}^{n-1} & \cdot & \left(\begin{array}{l}
1 \\
1
\end{array}\right) \hat{\alpha} & \hat{\chi}_{n+1}
\end{array}\right]+\left[\begin{array}{c}
a_{1} \\
a_{2} \\
a_{n} \\
b
\end{array}\right]+\left[\begin{array}{c}
\left(\begin{array}{c}
n+1 \\
1
\end{array}\right) \hat{\alpha} \\
\left(\begin{array}{c}
n+1 \\
2
\end{array}\right) \hat{\alpha}^{2} \\
\cdot \\
\left(\begin{array}{c}
n+1 \\
n
\end{array}\right) \hat{\alpha}^{n} \\
\left(\begin{array}{l}
n+1 \\
n+1
\end{array}\right) \hat{\alpha}^{n+1}
\end{array}\right],
$$

where

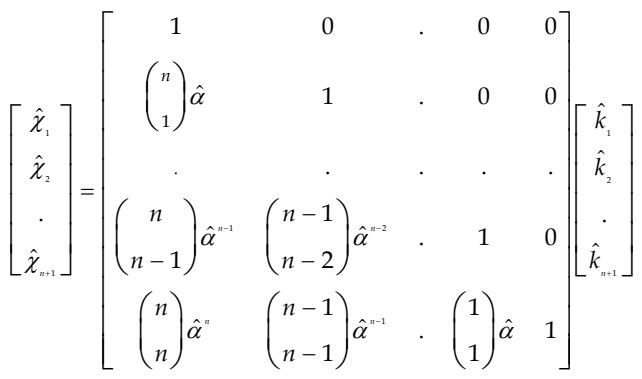

Proof. The proof is obtained by making standard manipulations and for brevity it has been omitted.

Now, as pre-announced, some preliminary results about the externally positive systems are stated.

Theorem 4. Connecting in series two or more SISO systems, linear, time-invariant and externally positive it is obtained another externally positive system.

Proof. If $W_{1}(s)$ and $W_{2}(s)$ are the transfer functions of two SISO externally positive systems then $w_{1}(t)=\mathcal{L}^{-1}\left(W_{1}(s)\right) \geq 0$ and $w_{2}(t)=\mathcal{L}^{-1}\left(W_{2}(s)\right) \geq 0$. From this and considering that

$$
w(t)=\mathcal{L}^{-1}\left(W_{1}(s) W_{2}(s)\right)=\int_{0}^{t} w_{1}(t-\tau) w_{2}(\tau) d \tau
$$

the proof follows.

Theorem 5. A third-order SISO linear and time-invariant system with transfer function

$$
W(s)=\frac{1}{(s-p)\left[(s-\alpha)^{2}+\omega^{2}\right]},
$$

i.e. without zeros, with a real pole $p$ and a couple of complex poles $\alpha \pm j \omega$, is externally positive iff $\alpha \leq p$, i.e. iff the real pole is not on the left of the couple of complex poles.

Proof. By using the translation property of the Laplace transform it is 


$$
w(t)=\mathcal{L}^{-1}\left(\frac{1}{(s-p)\left[(s-\alpha)^{2}+\omega^{2}\right]}\right)=e^{p t} \mathcal{L}^{-1}\left(\frac{1}{s\left[(s-\alpha+p)^{2}+\omega^{2}\right]}\right)=e^{p t} \int_{0}^{t} e^{(\alpha-p) \tau} \sin \omega \tau d \tau .
$$

Note that the signal $v(t)=e^{(\alpha-p) t} \sin \omega t$ is composed by a succession of positive and negative alternately waves. Therefore the integral $v_{i}(t)$ of this signal is non negative iff the succession of the absolute values of the areas of the considered semi-waves is non decreasing. Clearly this fact occurs iff the factor $e^{(\alpha-p) t}$ is non increasing, i.e. iff $\alpha-p \leq 0$, from which the proof derives.

From Theorems 4 and 5 easily follows that:

- a SISO system with a transfer function without zeros and all the poles real is externally positive;

- a SISO system with a transfer function without zeros and at least a real pole not on the left of every couple of complex poles is externally positive.

By using the above proposed results the following main results can be stated.

\section{First main result}

The following main result, useful to design a robust controller satisfying the required specifications 1, holds.

Theorem 6. Give the process (3) with limited uncertainties, a set of reference poles $\bar{P}$ and some design values $\hat{K}_{v}$ and $\hat{\tau}_{\max }$. If it is chosen $\bar{b}=b^{-}$and $\bar{a}_{n}=a_{n}^{+}$then $\forall \hat{\rho} \geq \hat{\rho}_{K}$, where

$$
\hat{\rho}_{K}=\hat{K}_{v} \frac{\bar{d}_{n}}{\bar{d}_{n+1}},
$$

the constant gain $K_{v}$ of the control system of Fig. 2, with a controller designed by using (21), is not minus than $\hat{K}_{v}, \forall a_{i} \in\left[a_{i}^{-}, a_{i}^{+}\right], i=1,2, \ldots, n$, and $\forall b \in\left[b^{-}, b^{+}\right]$. Moreover, by choosing the poles $\bar{P}$ all in -1 or of Bessel or of Butterworth, for $\hat{\rho} \gg \hat{\rho}_{\tau}$, where

$$
\hat{\rho}_{\tau}=-\frac{1}{\hat{\tau}_{\max } \max \operatorname{Real}(\bar{P})}
$$

the polynomial $d(s-\hat{\alpha})$ given by (27) is hurwitzian $\forall a_{i} \in\left[a_{i}^{-}, a_{i}^{+}\right], i=1,2, \ldots, n$, and $\forall b \in\left[b^{-}, b^{+}\right]$.

Proof. The proof of the first part of the theorem easily follows from (23) and from the fact that $\bar{b}=b^{-}$and $\bar{a}_{n}=a_{n}^{+}$.

In order to prove the second part of the theorem note that, from (22), (24), (25) and (26), for $\hat{\rho} \gg \hat{\rho}_{\tau}$ it is $d(s) \cong \tilde{d}(s)=\hat{d}(s)+h \hat{n}(s), h \geq 0$. Since for $\Delta b=0(\Leftrightarrow h=0)$ the roots of $\tilde{d}(s)$ are equal to the ones of $\hat{d}(s)$ and the zeros of $\hat{n}(s)$ are always on the right of the roots of $\hat{d}(s)$ and on the left of the imaginary axis (see Figs. 3, 4; from Fig. 4 it is possible to note that if the poles $\bar{P}$ are all in -1 then the zeros of $\hat{n}(s)$ have real part equal to $-\hat{\rho} / 2)$, it is that the root locus of $\tilde{d}(s)$ has a negative real asymptote and $n$ branches which go to the roots of $\hat{n}(s)$. From this consideration the second part of the proof follows.

From Theorems 3 and 6 several algorithms to design a controller such that $\forall a_{i} \in\left[a_{i}^{-}, a_{i}^{+}\right], i=1,2, \ldots, n$, and $\forall b \in\left[b^{-}, b^{+}\right]$the controlled system of Fig. 2 is of type one, 
with constant gain $K_{v} \geq \hat{K}_{v}$ and maximum time constant $\tau_{\max } \leq \hat{\tau}_{\max }$, where $\hat{K}_{v}$ and $\hat{\tau}_{\max }$ are design specifications (robustness of the constant gain and of the maximum time constant with respect to the parametric uncertainties of the process).

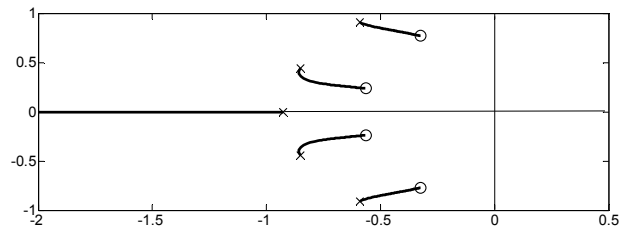

Fig. 3. Root locus of $\tilde{d}(s)$, Bessel poles, $n_{c}=n+1=5$ and $\rho=1$.

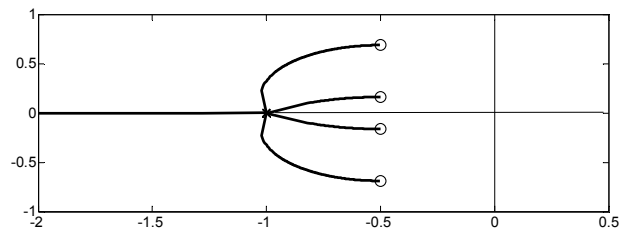

Fig. 4. Root locus of $\tilde{d}(s)$, coincident poles, $n_{c}=n+1=5$ and $\rho=1$.

A very simple algorithm is the following.

\section{Algorithm 1}

Step 1. By using (33) and (34), $\hat{\rho}=\max \left\{\hat{\rho}_{K}, \hat{\rho}_{\tau}\right\}$ is obtained and by using (21) the gains $\hat{k}_{i}, i=1, \ldots, n+1$ are computed.

Step 2. $\hat{\rho}$ is iteratively increased, if necessary, until by using (28) and Kharitonov's theorem, the polynomial $d(s-\hat{\alpha})$ given by (27) becomes hurwitzian $\forall a_{i} \in\left[a_{i}^{-}, a_{i}^{+}\right], i=1,2, \ldots, n$, and $\forall b \in\left[b^{-}, b^{+}\right]$. If the only uncertain parameter is $b$ (e.g. because of the uncertainty of the gain $g_{a}$ of the "power" actuator), instead of using Kharitonov's theorem it can be directly plot the root locus of $d(s)$ with respect to $b$.

Remark 6. Note that, if the uncertainties of the process are small enough and $\hat{\rho}$ is chosen big enough, it is $d(s) \cong \hat{d}(s)$. Therefore, by using Theorem 1 , turns out to be: $s \cong \bar{s}$, $t_{s} \cong \frac{\bar{t}_{s}}{\hat{\rho}}, \quad t_{a} \cong \frac{\bar{t}_{a}}{\hat{\rho}}, \quad \omega_{s}=\hat{\rho} \bar{\omega}_{s}, K_{v} \cong \hat{\rho} \bar{K}_{v}$. Moreover, if the poles $\bar{P}$ are equal to -1 or are of Bessel or of Butterworth, the values of $\bar{s}, \bar{t}_{s}, \bar{t}_{a}, \bar{\omega}_{s}, \bar{K}_{v}$ (intensively studied in the optimization theory) are well-known and/or easily computing (Butterworth, 1930), (Paarmann, 2001).

\section{Second main result}

The following fundamental result, that is the key to design a robust controller satisfying the required specifications 2., is stated. 
Theorem 7. Consider the process (3) with limited uncertainties and assigned design values of $\hat{K}_{v}$ and $\hat{\delta}_{\dot{r}-\dot{d}}$. If there exist a set of reference poles $\bar{P}$ and a $\hat{\rho}$ such that, with $\hat{k}_{j}, \mathrm{j}=1,2, \ldots, n+1$, provided by (21), $\forall a_{i} \in\left[a_{i}^{-}, a_{i}^{+}\right], \quad i=1,2, \ldots, n$, and $\forall b \in\left[b^{-}, b^{+}\right]$the transfer function

$$
W(s)=\frac{d_{n+1}}{s^{n+1}+d_{1} s^{n}+\ldots+d_{n} s+d_{n+1}}=\frac{b \hat{k}_{n+1}}{s^{n+1}+\left(a_{1}+b \hat{k}_{1}\right) s^{n}+\ldots+\left(a_{n}+b \hat{k}_{n}\right) s+b \hat{k}_{n+1}}
$$

is strictly hurwitzian and externally positive and $K_{v}=d_{n+1} / d_{n} \geq \hat{K}_{v}$, then, in the hypothesis that the initial state of the control system of Fig. 2 with $k_{i}=\hat{k}_{i}$ is null and that $r(0)-d(0)=0$, the corresponding tracking error $e(t)$, always $\forall a_{i} \in\left[a_{i}^{-}, a_{i}^{+}\right], i=1,2, \ldots, n$, and $\forall b \in\left[b^{-}, b^{+}\right]$, satisfies relation

$$
|e(t)| \leq \frac{1}{\hat{K}_{v}} \hat{\delta}_{\dot{r}-\dot{d}}, \quad \forall t \geq 0, \quad \forall r(t), d(t): \delta_{\dot{r}-\dot{d}}=\max _{\sigma \in[0, t]}|\dot{r}(\sigma)-\dot{d}(\sigma)| \leq \hat{\delta}_{\dot{r}-\dot{d}} .
$$

Moreover the overshoot $s$ is always null.

Proof. Note that the function $S_{p}(s)$ given by (11) is

$$
S_{p}(s)=\frac{1}{s}(1-W(s)) .
$$

Hence

$$
s_{p}(t)=1-w_{-1}(t)
$$

Since, for hypothesis, $w(t)$ is non negative then $w_{-1}(t)=\int^{t} w(\tau) d \tau$ is non decreasing with a final value $\left.W(s)\right|_{s=0}=1$. Therefore $s_{p}(t)$ is surely non negative. From this, by taking into account (7), (13) and (14), it follows that

$$
H_{v}=\frac{1}{\int_{0}^{\infty}\left|s_{p}(\tau)\right| d \tau}=\frac{1}{\int_{0}^{\infty} s_{p}(\tau) d \tau}=\frac{1}{\left.S_{p}(s)\right|_{s=0}}=\frac{1}{d_{n} / d_{n+1}}=\frac{d_{n+1}}{d_{n}}=K_{v} \geq \hat{K}_{v}
$$

and hence the proof.

Remark 7. The choice of $\bar{P}$ and the determination of a $\hat{\rho}$ such that (36) is valid, if the uncertainties of the process are null, are very simple. Indeed, by using Theorems 4 and 5 , it is sufficient to choose $\bar{P}$ with all the poles real or with at least a real pole not on the left of each couple of complex poles (e.g. $\bar{P}=\{-1,-1\}, \bar{P}=\{-1,-1+i,-1-i\}$, $\bar{P}=\{-1,-1,-1+i,-1-i\}, \ldots)$ and then to compute $\hat{\rho}$ by using relation $\hat{\rho}=\hat{K}_{v} \bar{d}_{n} / \bar{d}_{n+1}$.

If the process has parametric uncertainties, it is intuitive that the choice of $\bar{P}$ can be made with at least a real pole dominant with respect to each couple of complex poles and then to go on by using the Theorems of Sturm and/or Kharitonov or with new results or directly with the command roots and with the Monte Carlo method.

Regarding this the following main theorem holds. 
Theorem 8. Give the process (3) with limited uncertainties and with assigned nominal values of its parameters. Suppose that there exists a set of reference poles $\bar{P}=\left\{\bar{p}_{1}, \bar{p}_{2}, \ldots, \bar{p}_{n+1}\right\}$ such that the system

$$
\bar{W}_{h}(s)=\frac{1}{\bar{d}(s)+h \bar{n}(s)}, \quad \bar{d}(s)=\prod_{i=1}^{n+1}\left(s-p_{i}\right)=s^{n+1}+\bar{d}_{1} s^{n}+\ldots+\bar{d}_{n} s+\bar{d}_{n+1}, \bar{n}(s)=\bar{d}(s)-s^{n+1},
$$

is externally positive $\forall h \geq 0$. Then for $\hat{\rho}$ big enough the control system of Fig. 2, with $k_{j}=\hat{k}_{j}, \mathrm{j}=1,2, \ldots, n+1$, given by (21), $\forall a_{i} \in\left[a_{i}^{-}, a_{i}^{+}\right], \quad i=1,2, \ldots, n$, and $\forall b \in\left[b^{-}, b^{+}\right]$is externally positive.

Proof. Note that, taking into account (22), (24), (25) and (26), for $\hat{\rho}$ big enough it is $d(s) \cong \tilde{d}(s)=\hat{d}(s)+h \hat{n}(s)$. From this the proof easily follows.

In the following, for brevity, the second, third, fourth-order control systems will be considered.

Theorem 9. Some sets of reference poles $\bar{P}$ which satisfy Theorem 8 are: $\bar{P}=\{-1,-\alpha\} / \sqrt{\alpha}$ with $\alpha>1$ (e.g. $\alpha=1.5,2, \ldots) ; \bar{P}=\{-1,-\alpha+i \omega,-\alpha-i \omega\} / \sqrt[3]{\alpha^{2}+\omega^{2}} \quad$ with $\alpha>1$ and $\omega$ such that the roots of $\bar{n}(s)$ are real (e.g. $a=-1.5$ and $\omega \geq 2.598, a=-2$ and $\omega \geq 2.544, \ldots) ; \bar{P}=\{-1,-\alpha,-\alpha,-\alpha\} / \sqrt[4]{\alpha^{3}}, \alpha>1$ (e.g. $\alpha=1.5,2, \ldots$ ).

Proof. The proof easily follows from the root loci of $\bar{d}(s)=\bar{d}(s)+h \bar{n}(s)$ (see Figs. 5, 6).

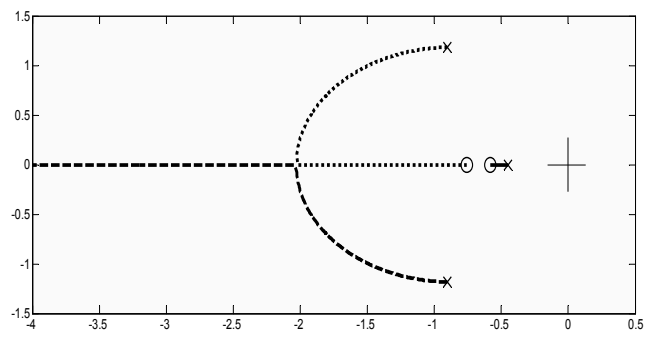

Fig. 5. Root locus of $\tilde{\bar{d}}(s), n_{c}=n+1=3$.

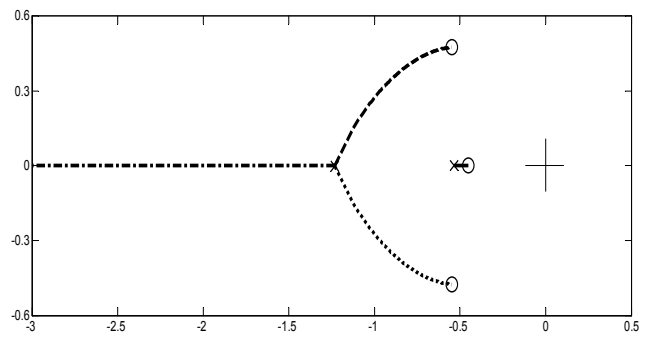

Fig. 6. Root locus of $\tilde{\bar{d}}(s), n_{c}=n+1=4$.

To verify the externally positivity of a third-order system the following theorems are useful. 
Theorem 10. Let be

$$
W(s)=\frac{d_{3}}{s^{3}+d_{1} s^{2}+d_{2} s+d_{3}}=\frac{d_{3}}{d(s)}
$$

an asimptotically stable system. If

$$
\delta=27 d\left(-d_{1} / 3\right)=2 d_{1}-9 d_{1} d_{2}+27 d_{3}<0
$$

then the poles of $W(s)$ are all real or the real pole is on the right of the remaining couple of complex poles, i.e. the system is externally positive.

Proof. Let be $p_{1}, p_{2}, p_{3}$ the poles of $W(s)$ note that the "barycentre" $x_{c}=-d_{1} / 3$ is in the interval $\left[\min \operatorname{Real}\left(p_{i}\right), \max \operatorname{Real}\left(p_{i}\right)\right]$. Hence if relation (42) is satisfied, as $d(0)=d_{3}>0$, the interval $\left[x_{c}, 0\right]$ contains a real pole (see Figs. 7, 8). From this the proof easily follows.

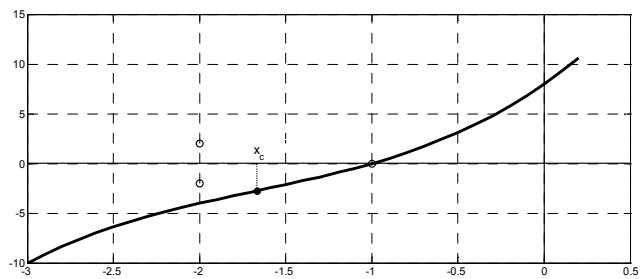

Fig. 7. $\delta$ in the case of real pole on the right of the couple of complex poles.

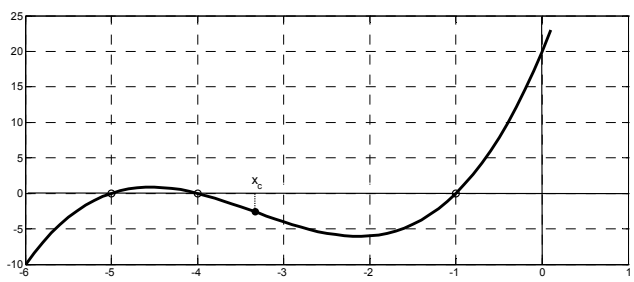

Fig. 8. $\delta$ in the case of all real poles.

Theorem 11. Give the control system

$$
W(s)=\frac{b \hat{k}_{3}}{s^{3}+\left(a_{1}+b \hat{k}_{1}\right) s^{2}+\left(a_{2}+b \hat{k}_{2}\right) s+b \hat{k}_{3}}, \quad a_{1} \in\left[a_{1}^{-}, a_{1}^{+}\right], a_{2} \in\left[a_{2}^{-}, a_{2}^{+}\right], b \in\left[b^{-}, b^{+}\right],
$$

if $\hat{k}_{1}, \hat{k}_{2}, \hat{k}_{3}$ satisfy the relations:

$$
\begin{aligned}
& b \hat{k}_{3}>0, \forall b \in\left[b^{-}, b^{+}\right] \\
& \delta\left(a_{1}, a_{2}^{-}, b\right)=2\left(a_{1}+b \hat{k}_{1}\right)^{3}-9\left(a_{1}+b \hat{k}_{1}\right)\left(a_{2}^{-}+b \hat{k}_{1}\right)+27 b \hat{k}_{3}<0, \forall b \in\left[b^{-}, b^{+}\right] \text {and } a_{1}=\left\{a_{1}^{-}, a_{1}^{+}\right\},
\end{aligned}
$$

then the control system is externally positive $\forall a_{1} \in\left[a_{1}^{-}, a_{1}^{+}\right], \forall a_{2} \in\left[a_{2}^{-}, a_{2}^{+}\right]$and $\forall b \in\left[b^{-}, b^{+}\right]$. 
Proof. Note that if $\delta\left(a_{1}, a_{2}^{-}, b\right)<0, \forall a_{1} \in\left[a_{1}^{-}, a_{1}^{+}\right]$and $\forall b \in\left[b^{-}, b^{+}\right]$, then $\delta\left(a_{1}, a_{2}, b\right)<0$, $\forall a_{1} \in\left[a_{1}^{-}, a_{1}^{+}\right], \forall a_{2} \in\left[a_{2}^{-}, a_{2}^{+}\right]$and $\forall b \in\left[b^{-}, b^{+}\right]$. Moreover if $\delta\left(a_{1}^{-}, a_{2}^{-}, b\right)<0$ and $\delta\left(a_{1}^{+}, a_{2}^{-}, b\right)<0, \forall b \in\left[b^{-}, b^{+}\right]$, then by using Theorem 10 the polynomials

$$
\begin{aligned}
& d^{-}(s)=s^{3}+\left(a_{1}^{-}+b \hat{k}_{1}\right) s^{2}+\left(a_{2}^{-}+b \hat{k}_{2}\right) s+b \hat{k}_{3} \\
& d^{+}(s)=s^{3}+\left(a_{1}^{+}+b \hat{k}_{1}\right) s^{2}+\left(a_{2}^{-}+b \hat{k}_{2}\right) s+b \hat{k}_{3},
\end{aligned}
$$

$\forall b \in\left[b^{-}, b^{+}\right]$, have a dominant real root. By taking into account the root loci with respect $h$ of the polynomial

$$
d(s)=s^{3}+\left(a_{1}^{-}+b \hat{k}_{1}\right) s^{2}+\left(a_{2}^{-}+b \hat{k}_{2}\right) s+b \hat{k}_{3}+h s^{2},
$$

in the two cases of polynomial $d^{-}(s)$ with all the roots real negative and of polynomial $d^{-}(s)$ with a real negative root on the right of the remaining complex roots (see Figs. 9, 10), the proof easily follows.

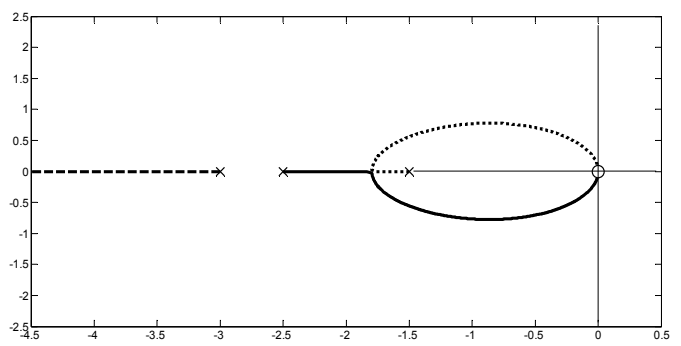

Fig. 9. Root locus of the polynomial (46) in the hypothesis that all the roots of $d^{-}(s)$ are real.

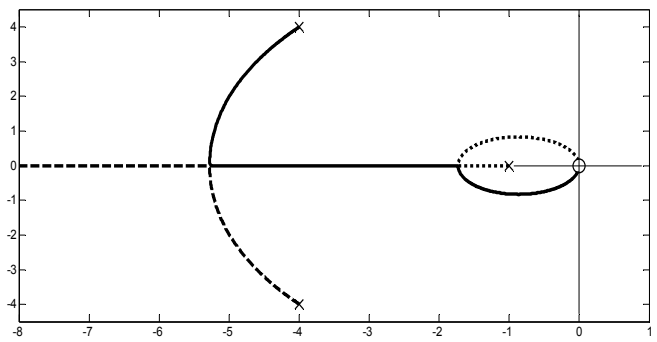

Fig. 10. Root locus of the polynomial (46) under the hypothesis that $d^{-}(s)$ has a real negative root on the right of the remaining complex roots.

Finally, from Theorems 7, 9, 11 and by using the Routh criterion the next theorem easily follows.

Theorem 12. Give the process (3) with limited uncertainties for $n_{c}=n+1=3$ and assigned some design values of $\hat{K}_{v}$ and $\hat{\delta}_{\dot{r}-\dot{d}}$. Let be choose $\bar{P}=\left\{\bar{p}_{1}, \bar{p}_{2}, \bar{p}_{2}\right\}=$ $\{-1,-\alpha+i \omega,-\alpha-i \omega\} / \sqrt[3]{\alpha^{2}+\omega^{2}}$, with $\alpha>1$ and $\omega$ such that the roots of 


$$
\bar{n}(s)=s^{3}-\bar{d}(s)=\bar{d}_{1} s^{2}+\bar{d}_{2} s+\bar{d}_{3}, \quad \bar{d}(s)=\left(s-\bar{p}_{1}\right)\left(s-\bar{p}_{2}\right)\left(s-\bar{p}_{3}\right)
$$

are real (e.g. $a=-1.5$ and $\omega \geq 2.598, a=-2$ and $\omega \geq 2.544, \ldots$ ). Then said $\hat{\rho}$ a number not minus than

$$
\hat{\rho}_{K}=\hat{K}_{v} \frac{\bar{d}_{n}}{\bar{d}_{n+1}},
$$

such that:

$$
\begin{aligned}
& \delta\left(a_{1}, a_{2}^{-}, b\right)=2\left(a_{1}+b \hat{k}_{1}\right)^{3}-9\left(a_{1}+b \hat{k}_{1}\right)\left(\left(a_{2}^{-}+b \hat{k}_{2}\right)\right)+27 b \hat{k}_{3}<0 \\
& \forall b \in\left[b^{-}, b^{+}\right] \text {and } a_{1}=\left\{a_{1}^{-}, a_{1}^{+}\right\} \\
& a_{1}^{-}+b^{-} \hat{k}_{1}>0, \quad \hat{k}_{1} \hat{k}_{2} b^{2}+b\left(\hat{k}_{1}+\hat{k}_{2}-\hat{k}_{3}\right)+a_{1}^{-} a_{2}^{-}>0, \forall b \in\left[b^{-}, b^{+}\right],
\end{aligned}
$$

where

$$
\hat{k}_{1}=\frac{\rho \bar{d}_{1}-a_{1}^{-}}{b^{-}}, \quad \hat{k}_{2}=\frac{\rho^{2} \bar{d}_{2}-a_{2}^{+}}{b^{-}}, \quad \hat{k}_{3}=\frac{\rho^{3} \bar{d}_{3}}{b^{-}}
$$

under the hypothesis that the initial state of the control system of Fig. 2, with $n_{c}=n+1=3$ and $k_{i}=\hat{k}_{i}$, is null and that $r(0)-d(0)=0$, the error $e(t)$ of the control system of Fig. 2, considering all the possible values of the process, satisfies relation

$$
|e(t)| \leq \frac{1}{\hat{K}_{v}} \hat{\delta}_{\dot{r}-\dot{d}}, \quad \forall t \geq 0, \quad \forall r(t), d(t): \delta_{\dot{r}-\dot{d}}=\max _{\sigma \in[0, t]}|\dot{r}(\sigma)-\dot{d}(\sigma)| \leq \hat{\delta}_{\dot{r}-\dot{d}} .
$$

Note that, by applying the Routh conditions (50) to the polynomial $d(s-\hat{\alpha}), \hat{\alpha}=1 / \hat{\tau}_{\max }$, instead of to $d(s)$, it is possible to satisfy also the specification about $\tau_{\max }$; so the specifications 2. are all satisfied.

Remark 8. Give the process (3) with limited uncertainties and assigne the design values of $\hat{K}_{v}, \hat{\tau}_{\max }$ and of $\hat{\delta}_{\dot{r}-\dot{d}}$; if $n_{c}=n+1=2,3,4$, by choosing $\bar{P}$ in accordance with Theorem 9, a controller such that, for all the possible values of the parameters of the process, $\tau_{\max } \leq \hat{\tau}_{\max }$ and the error $e(t)$ satisfies relation (2), can be obtained by increasing, if necessary, iteratively $\hat{\rho}$ starting from the value of $\hat{\rho}_{K}=\hat{K}_{v} \bar{d}_{n} / \bar{d}_{n+1}$ with the help of the command roots and with the Monte Carlo method.

According to this, note that for $n_{c} \leq 4$ the control system of Fig. 2 (for an assigned set of parameters) is externally positive and $\tau_{\max } \leq \hat{\tau}_{\max }$ if, denoting with $p_{j}$ the root of $d(s)$ having the maximum real part, $\operatorname{imag}\left(p_{j}\right)=0$ and $\operatorname{real}\left(p_{j}\right) \leq-1 / \hat{\tau}_{\max }$.

Note that the proposed design method, by taking into account Theorem 8, can be easily extended in the case of $n_{c} \geq 4$.

Example 1. Consider a planar robot (e.g. a plotter) whose end-effector must plot dashed linear and continuous lines with constant velocities during each line.

Under the hypothesis that each activation system is an electric DC motor (with inertial load, possible resistance in series and negligble inductance of armature) powered by using a power amplifier, the model of the process turns out to be 


$$
G(s)=\frac{b}{s^{2}+a_{1} s+a_{2}}, \quad a_{1}=\frac{R K_{a}+K^{2}}{R I}, a_{2}=0, b=\frac{K}{R I} g_{a} .
$$

If

$$
R=2.5 \pm 5 \%, K=0.5 \pm 5 \%, K_{a}=0.01 \pm 5 \%, I=0.05 \pm 5 \%, g_{a}=100 \pm 10 \% \text {, }
$$

it is

$$
1.8 \leq a_{1} \leq 2.7, \quad a_{2}=0, \quad 310 \leq b \leq 512 .
$$

By choosing $\bar{P}=\{-1,-\alpha+i \omega,-\alpha-i \omega\} / \sqrt[3]{\alpha^{2}+\omega^{2}}, \quad a=-1.5 \quad$ and $\quad \omega=2.598$, $a_{1 n}=2.25, b_{n}=310$, for $\hat{K}_{v}=2$ it is: $\hat{\rho}_{K}=5.547, \hat{k}_{1}=0.0271, \quad \hat{k}_{2}=0.275, \hat{k}_{3}=0.550$, $\max _{b} \delta\left(a_{1}^{-}, a_{2}^{-}, b\right)=-1.108 \mathrm{e} 3<0, \max _{b} \delta\left(a_{1}^{+}, a_{2}^{-}, b\right)=-1.181 \mathrm{e} 3<0, \tau_{\max } \leq 383 \mathrm{~ms}$.

Hence the controlled process is externally positive $\forall a_{1} \in[1.8,2.7]$ and $\forall b \in[310,512]$. Therefore the overshoot is always null; moreover, said $r_{x}, r_{y}$ the components of the reference trajectory of the controlled robot, the corresponding tracking errors satisfy relations $\left|e_{x}\right| \leq\left|\dot{r}_{x}\right| / 2$ and $\left|e_{y}\right| \leq\left|\dot{r}_{y}\right| / 2$.

For $\hat{K}_{v}=10$ it is obtained that: $\hat{\rho}_{K}=27.734, \hat{k}_{1}=0.165, \hat{k}_{2}=6.877, \hat{k}_{3}=68.771$, $\max _{b} \delta\left(a_{1}^{-}, a_{2}^{-}, b\right)=-1.436 \mathrm{e} 5<0, \quad \max _{b} \delta\left(a_{1}^{+}, a_{2}^{-}, b\right)=-1.454 \mathrm{e} 5<0, \tau_{\max } \leq 75.3 \mathrm{~ms}$.

Hence $\left|e_{x}\right| \leq\left|\dot{r}_{x}\right| / 10$ and $\left|e_{y}\right| \leq\left|\dot{r}_{y}\right| / 10$.

Suppose that a tracking goal is to engrave on a big board of size $2.5 \times 0.70 \mathrm{~m}^{2}$ the word INTECH (see Fig. 12 ). In Fig. 11 the time histories of $r_{x}, \dot{r}_{x}$ and, under the hypothesis that $\hat{K}_{v}=2$, the corresponding error $e_{x}$, in accordance with the proposed results are reported. Clearly the "tracking precision" is unchanged $\forall r_{x}$ with the same maximum value of $\left|\dot{r}_{x}\right|$. Figs. 13 and 14 show the engraved words for $\hat{K}_{v}=2$ and $\hat{K}_{v}=10$, respectively.
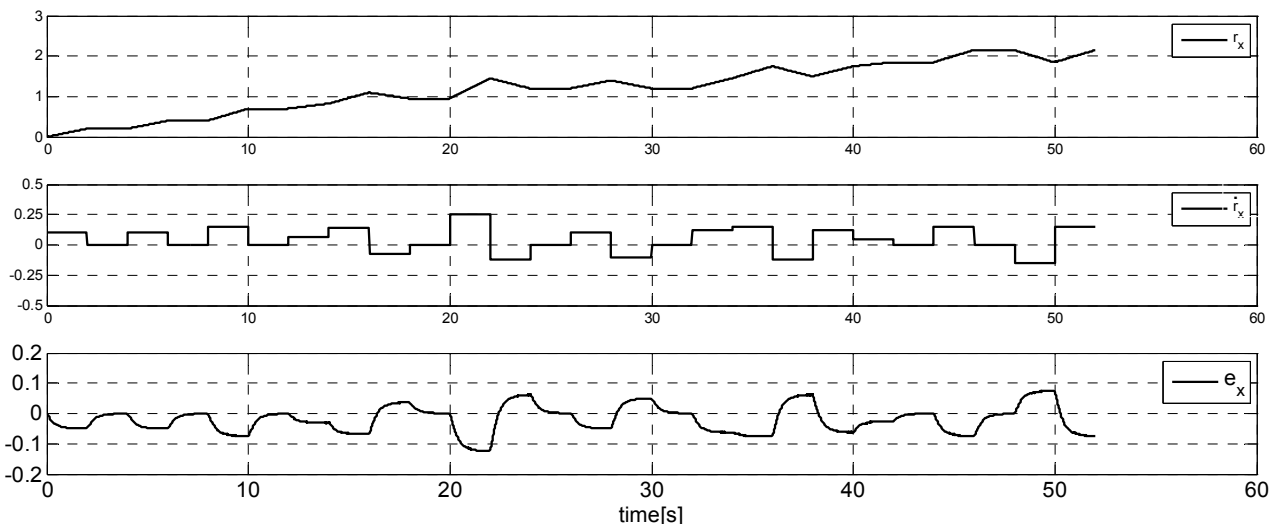

Fig. 11. Time histories of $r_{x}, \dot{r}_{x}$ and $e_{x}$ for $\hat{K}_{v}=2$. 


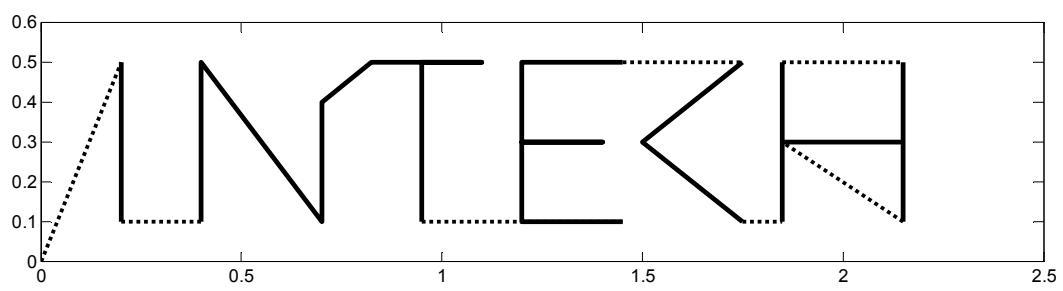

Fig. 12. The desired "word".

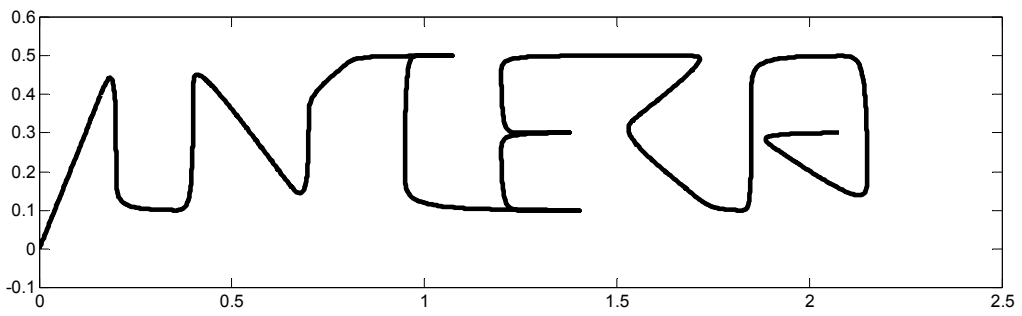

Fig. 13. The engraved word with $\hat{K}_{v}=2$.

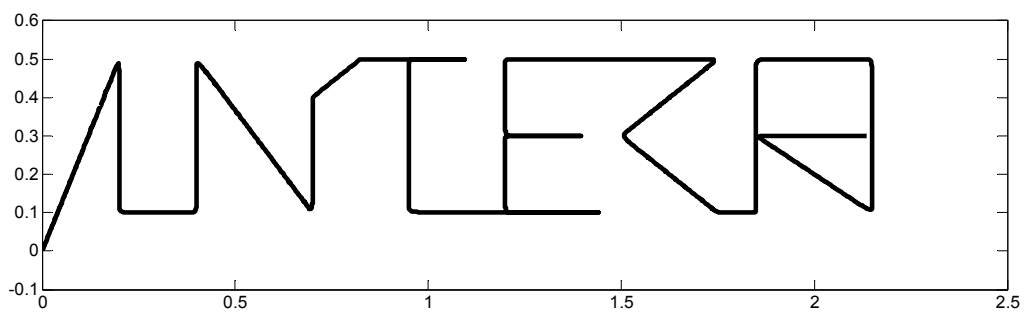

Fig. 14. The engraved word with $\hat{K}_{v}=10$.

\section{Part II}

\section{Problem formulation and preliminary results}

Now consider the following class of nonlinear dynamic system

$$
\ddot{y}=F_{1}(y, \dot{y}, p) u+F_{2}(y, \dot{y}, p) \dot{y}+f(t, y, \dot{y}, p), \quad F_{2}(y, \dot{y}, p)=\sum_{i=1}^{m} F_{2 i}(y, \dot{y}, p) \dot{y}_{i},
$$

where $t \in R$ is the time, $y \in R^{m}$ is the output, $u \in R^{r}$ is the control input, $p \in \wp \subset R^{\mu}$ is the uncertain parametric vector of the system, with $\wp$ a compact set, $F_{1} \in R^{m \times r}$ is a limited matrix with rank $m, F_{2 i} \in R^{m x m}$ are limited matrices and $f \in R^{m}$ is a limited vector which models possible disturbances and/or particular nonlinearities of the system.

In the following it is supposed that there exists at least a matrix $K(y, \dot{y}) \in R^{r \times m}$ such that the matrix $H=F_{1} K$ is positive definite (p.d.) $\forall p \in \wp$. 
Remark 9. It is important to note that the class of systems (56) includes the one, very important from a practical point of view, of the articulated mechanical systems (mechanical structures, flexible too, robots,...). Indeed it is well-known that mechanical systems can be described as follows

where:

$$
B \ddot{q}=c+g+T u,
$$

- $\quad q \in R^{m}$ is the vector of the Lagrangian coordinates,

- $B(q, p)$ is the inertia matrix (p.d.), in which $p \in \wp \subset R^{\mu}$, with $\wp$ a compact set, is the vector of the uncertain parameters of the mechanical system,

- $\quad c=C(q, \dot{q}, p) \dot{q}$, with $C$ linear with respect to $\dot{q}$, is the vector of the generalized centrifugal forces, the Coriolis and friction ones,

- $g=g(t, q, p)$ is the vector of the generalized gravitational and elastic forces and of the external disturbances,

- $\quad u$ is the vector of the generalized control forces produced by the actuators,

- $\quad T$ is the transmission matrix of the generalized control forces.

If system (56) is controlled by using the following state feedback control law with a partial compensation

$$
u=-K\left(K_{p} y+K_{d} \dot{y}\right)-u_{c},
$$

where $K_{p}, K_{d} \in R^{m \times m}$ are constant matrices, $K \in R^{r \times m}$ is a matrix depending in general on $t, y, \dot{y}$ and $u_{c}$ is the partial compensation signal, the closed-loop system is

$$
\begin{aligned}
& \dot{x}=\left[\begin{array}{cc}
0 & I \\
-H K_{p} & -H K_{d}
\end{array}\right] x+\left(\sum_{i=1}^{m}\left[\begin{array}{cc}
0 & 0 \\
0 & F_{2 i}
\end{array}\right] x_{m-1+i}\right) x+\left[\begin{array}{l}
0 \\
I
\end{array}\right] w=A_{1} x+\left(\sum_{i=1}^{m} A_{2 i} x_{m-1+i}\right) x+B w, \\
& \text { where } H=F_{1} K, \quad w=f-F_{1} u_{c} \\
& y=\left[\begin{array}{ll}
I & 0
\end{array}\right] x=C x, \quad \dot{y}=\left[\begin{array}{ll}
0 & I
\end{array}\right] x=C_{\dot{y}} x .
\end{aligned}
$$

In order to develop a practical robust stabilization method for system (59) the following definition and results are necessary.

Definition 2. Give system (59) and a symmetric p.d. matrix $P \in R^{n x n}$. A positive first-order system $\dot{\rho}=f(\rho, d), v=\eta(\rho), v_{p}=\eta_{p}(\rho)$, where $\rho=\|x\|_{P}=\sqrt{x^{T} P x}$ and $d=\max \|w\|$, such that $\|y\| \leq v,\|\dot{y}\| \leq v_{p}$ is said to be majorant system of system (59).

Theorem 13. Consider the quadratic system

$$
\dot{\rho}=\alpha_{1} \rho+\alpha_{2} \rho^{2}+\beta d=\alpha_{2} \rho^{2}+\alpha_{1} \rho+\alpha_{0}, \quad \alpha_{1}<0, \alpha_{2}, \beta \geq 0, \quad \rho(0)=\rho_{0} \geq 0, \quad d \geq 0 .
$$

If $\alpha_{1}^{2}-4 \alpha_{2} \beta d>0$ it is:

$$
\rho(t)=\frac{\rho_{1}-\rho_{2} \varphi(t)}{1-\varphi(t)}, \text { where } \varphi(t)=\frac{\rho_{0}-\rho_{1}}{\rho_{0}-\rho_{2}} e^{-t / \tau}, \tau=\frac{1}{\alpha_{2}\left(\rho_{2}-\rho_{1}\right)}, \lim _{t \rightarrow \infty} \rho(t) \leq \rho_{1}, \forall \rho_{0}<\rho_{2},
$$


where $\rho_{1}, \rho_{2}, \rho_{1}<\rho_{2}$, are the roots of the algebraic equation $\alpha_{2} \rho^{2}+\alpha_{1} \rho+\alpha_{0}=0$ (see Fig. 15). Moreover for $d=0$ the practical convergence time $t_{5 \%} \triangleq \rho\left(t_{5 \%}\right)=5 \% \rho_{0}$ is given by (see Fig. 16):

$$
t_{5 \%}=\gamma \tau_{l}, \tau_{l}=-1 / \alpha_{1}, \gamma=\ln \frac{20-\rho_{0} / \rho_{20}}{1-\rho_{0} / \rho_{20}}, \rho_{20}=-\alpha_{1} / \alpha_{2}
$$

in which $\tau_{l}$ is the time constant of the linearized of system (60) and $\rho_{20}$ is the upper bound of the convergence interval of $\rho(t)$ for $d=0$, i.e. of system (60) in free evolution.

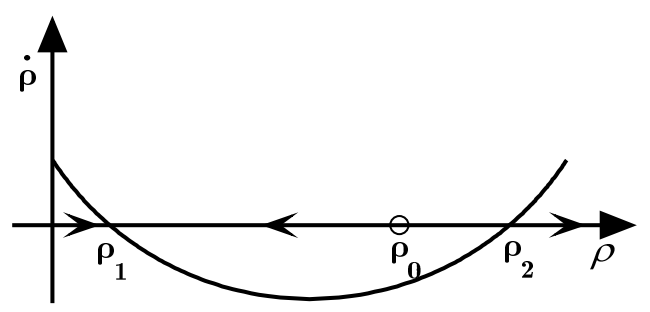

Fig. 15. Graphical representation of system(60).

Proof. The proof of (61) easily follows by solving, with the use of the method of separation of variables, the equation $d \rho / d t=\alpha_{2}\left(\rho-\rho_{1}\right)\left(\rho-\rho_{2}\right)$ and from Fig. 15. Instead (62) easily derives by noting that the solution of (60) for $d=0$ is

$$
\frac{\rho(t)}{\rho_{0}}=\frac{\rho_{20}}{\rho_{0}} \frac{1}{1+\left(\frac{\rho_{20}}{\rho_{0}}-1\right) e^{t / \tau l}} .
$$
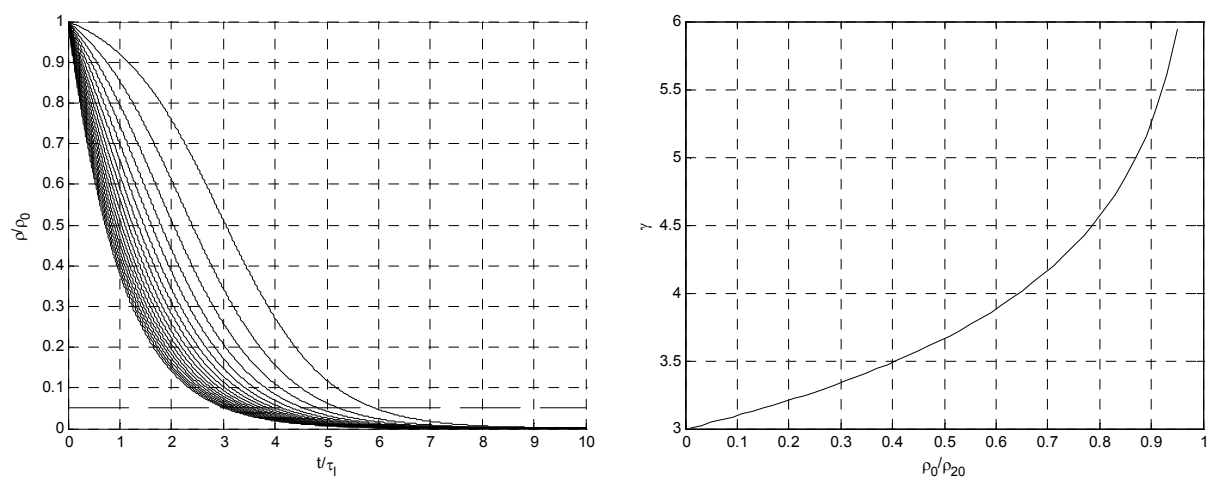

Fig. 16. Time history of $\rho$ and $\gamma$ as a function of $\rho_{0}$ 
Theorem 14. Give a matrix $P \in R^{n \times n} p . d$. and a matrix $C \in R^{m \times n}$ with rank $m$. If $\|x\|_{P} \leq \rho$ then the smallest $\alpha$ such that $\|v\| \leq \alpha\|x\|_{P} \leq \alpha \rho$, where $v=C x$, turns out to be $\alpha=\sqrt{\lambda_{\max }\left(C P^{-1} C^{T}\right)}$.

Proof. The proof is standard.

Theorem 15. Let be

$$
A=\sum_{i_{1}, i_{2}, \ldots, i_{\mu} \in\{0,1\}} A_{i_{1} i_{2} \ldots i_{\mu}} g_{1}\left(\pi_{1}\right)^{i_{1}} g_{2}\left(\pi_{2}\right)^{i_{2}} \ldots g_{\mu}\left(\pi_{\mu}\right)^{i_{\mu}} \in R^{m \times m}
$$

a symmetric matrix, where

$$
\left[\begin{array}{llll}
\pi_{1} & \pi_{2} & \ldots & \pi_{\mu}
\end{array}\right]^{T}=\pi \in \Pi=\left\{\pi \in R^{\mu}: \pi^{-} \leq \pi \leq \pi^{+}\right\}
$$

and each function $g_{i}, i=1, \ldots, \mu$, is continuous with respect to its argument, and $P \in R^{n \times n}$ a symmetric p.d. matrix. Then the minimum (maximum) of $\underset{\pi \in \Pi}{\lambda_{\min }}\left(Q P^{-1}\right)\left(\lambda_{\max }\left(Q P^{-1}\right)\right)$, where $Q=-\left(A^{T} P+P A\right):$ is assumed in one of the $2^{\mu}$ vertices of $\Gamma$, in which

$$
\Gamma=\left\{\gamma \in R^{\mu}: \min \left[g_{1} \ldots g_{\mu}\right] \leq \gamma \leq \max \left[g_{1} \ldots g_{\mu}\right]\right\} .
$$

Proof. The proof can be found in (Celentano, 2010).

\section{Main results}

Now the following main result, which provides a majorant system of the considered control system, is stated.

Theorem 16. Give a symmetric $p . d$. matrix $P \in R^{n \times n}$. Then a majorant system of the system (59) is

$$
\dot{\rho}=\alpha_{1} \rho+\alpha_{2} \rho^{2}+\beta d, v=c \rho, \quad v_{p}=c_{p} \rho,
$$

in which:

$$
\begin{gathered}
\alpha_{1}=-\min _{x \in C_{P, \rho}, p \in \wp} \frac{\lambda_{\min }\left(Q_{1} P^{-1}\right)}{2}, Q_{1}=-\left(A_{1}{ }^{T} P+P A_{1}\right) \\
\alpha_{2}=-\min _{x \in C_{P, 1}, p \in \wp} \frac{\lambda_{\min }\left(\sum_{i=1}^{m} Q_{2 i} P^{-1} x_{m+i-1}\right)}{2}, Q_{2 i}=-\left(A_{2 i}{ }^{T} P+P A_{2 i}\right) \\
\beta=\sqrt{\lambda_{\max }\left(B^{T} P B\right)}, c=\sqrt{\lambda_{\max }\left(C P^{-1} C^{T}\right)}, \quad c_{p}=\sqrt{\lambda_{\max }\left(C_{\dot{y}} P^{-1} C_{\dot{y}}^{T}\right)}, \quad d=\max _{t \in R, x \in C_{P, \rho}, p \in \wp}\|w\|,(70)
\end{gathered}
$$

where $C_{P, \rho}=\left\{x: x^{T} P x=\rho^{2}\right\}$.

Proof. By choosing as "Lyapunov function" the quadratic form $V=x^{T} P x=\|x\|_{P}^{2}=\rho^{2}$, for $x$ belonging to a generic hyper-ellipse $C_{P, \rho}$, it is 


$$
\dot{\rho} \leq-\min _{x \in C_{P, \rho}, p \in \wp} \frac{x^{T} Q_{1} x}{2 x^{T} P x}-\min _{x \in C_{P, \rho}, p \in \wp} \frac{x^{T} \sum_{i=1}^{n} Q_{2 i} x_{i} P^{-1} x}{2 x^{T} P x}+\max _{t, x \in C_{P, \rho}, p \in \wp} \frac{x^{T} P B w}{x^{T} P x} .
$$

The proof easily follows from (71)

It is valid the following important "non-interaction" theorem.

Theorem 17. If in Theorem 16 it is

$$
K_{p}=I a^{2}, \quad K_{d}=\sqrt{2} I a, \quad P=\left[\begin{array}{cc}
\sqrt{2} a I & I \\
I & \frac{\sqrt{2}}{a} I
\end{array}\right], \quad a>0,
$$

then:

$$
\begin{gathered}
\alpha_{1}=-\min _{x \in C_{P, \rho}, p \in \wp,} \frac{\lambda_{\min }\left(Q_{1} P^{-1}\right)}{2}=\left\{\begin{array}{cc}
-\frac{a}{\sqrt{2}}\left[\lambda_{\min }\left(H^{T}+H\right)-1\right], & \text { if } \lambda_{\min }\left(H^{T}+H\right)<2 \\
-\frac{a}{\sqrt{2}}, & \text { if } \lambda_{\min }\left(H^{T}+H\right) \geq 2,
\end{array}\right. \\
\alpha_{2}=-\min _{x \in C_{P, 1}, p \in \wp} \frac{\lambda_{\min }\left(\sum_{i=1}^{m}\left[\begin{array}{cc}
A_{2 i} & -\sqrt{2} A_{2 i} \\
\sqrt{2} A_{2 i} & -2 A_{2 i}-A_{2 i}^{T}
\end{array}\right] x_{m+i-1}\right)}{2}, \\
\beta=\frac{\sqrt[4]{2}, \quad c=\frac{\sqrt[4]{2}}{\sqrt{a},} \quad c_{p}=\sqrt[4]{2} \sqrt{a} .}{}
\end{gathered}
$$

Proof. First note that, by making the change of variable $z=T^{-1} x$, with $T$ such that $z=\left[\begin{array}{lllllll}y_{1} & \dot{y}_{1} & y_{2} & \dot{y}_{2} & \ldots & y_{m} & \dot{y}_{m}\end{array}\right]^{T}$, the matrix $\hat{P}=T^{T} P T$ is block-diagonal 'with blocks on the principal diagonal equal to

$$
\hat{P}_{i i}=\left[\begin{array}{cc}
\sqrt{2} a & 1 \\
1 & \frac{\sqrt{2}}{a}
\end{array}\right] .
$$

Since $\hat{P}_{i i}$ is p.d. $\forall a>0$, it follows that $\hat{P}$ is $p . d$. and, therefore, also $P$ is p.d. .

Now note that

hence

$$
\left[\begin{array}{cc}
\sqrt{2} a I & I \\
I & \frac{\sqrt{2}}{a} I
\end{array}\right]\left[\begin{array}{cc}
\frac{\sqrt{2}}{a} I & -I \\
-I & \sqrt{2} a I
\end{array}\right]=\left[\begin{array}{cc}
I & 0 \\
0 & I
\end{array}\right]
$$

$$
P^{-1}=\left[\begin{array}{cc}
\frac{\sqrt{2}}{a} I & -I \\
-I & \sqrt{2} a I
\end{array}\right] .
$$


Then it is

Therefore

$$
\begin{aligned}
& Q P^{-1}=-\left(A_{1}^{T} P+P A_{1}\right) P^{-1}=-A_{1}^{T}-P A_{1} P^{-1}= \\
& =\left[\begin{array}{cc}
0 & a^{2} H^{T} \\
-I & \sqrt{2} a H^{T}
\end{array}\right]+\left[\begin{array}{cc}
\sqrt{2} a I & I \\
I & \frac{\sqrt{2}}{a} I
\end{array}\right]\left[\begin{array}{cc}
0 & -I \\
a^{2} H & \sqrt{2} a H
\end{array}\right]\left[\begin{array}{cc}
\frac{\sqrt{2}}{a} I & -I \\
-I & \sqrt{2} a I
\end{array}\right]= \\
& =\left[\begin{array}{cc}
0 & a^{2} H^{T} \\
-I & \sqrt{2} a H^{T}
\end{array}\right]+\left[\begin{array}{cc}
\sqrt{2} a I & I \\
I & \frac{\sqrt{2}}{a} I
\end{array}\right]\left[\begin{array}{cc}
I & -\sqrt{2} a I \\
0 & a^{2} H
\end{array}\right]= \\
& =\left[\begin{array}{cc}
0 & a^{2} H^{T} \\
-I & \sqrt{2} a H^{T}
\end{array}\right]+\left[\begin{array}{cc}
\sqrt{2} a I & a^{2}(H-2 I) \\
I & \sqrt{2} a(H-I)
\end{array}\right]=\left[\begin{array}{cc}
\sqrt{2} a I & a^{2}\left(H^{T}+H-2 I\right) \\
0 & \sqrt{2} a\left(H^{T}+H-I\right)
\end{array}\right] .
\end{aligned}
$$

from which (73) easily follows.

$$
\lambda\left(Q P^{-1}\right)=\lambda(\sqrt{2} a I) \cup \lambda\left(\sqrt{2} a\left(H^{T}+H-I\right)\right),
$$

In order to prove (74) note that, if $T$ is a symmetric nonsingular matrix, it is

$$
\lambda\left(Q_{2 i} P^{-1}\right)=\lambda\left(T Q_{2 i} P^{-1} T^{-1}\right)=\lambda\left(-\left(\hat{A}_{2 i}{ }^{T} \hat{P}+\hat{P} \hat{A}_{2 i}\right) \hat{P}^{-1}\right), \hat{A}_{2 i}=T A_{2 i} T^{-1}, \hat{P}=T^{-1} P T^{-1} .
$$

By choosing a matrix $T=\left[\begin{array}{cc}a I & 0 \\ 0 & I\end{array}\right]$ it is:

$$
\begin{aligned}
& \hat{A}_{2 i}=\left[\begin{array}{cc}
a I & 0 \\
0 & I
\end{array}\right]\left[\begin{array}{cc}
0 & 0 \\
0 & F_{2 i}
\end{array}\right]\left[\begin{array}{cc}
\frac{I}{a} & 0 \\
0 & I
\end{array}\right]=\left[\begin{array}{cc}
0 & 0 \\
0 & F_{2 i}
\end{array}\right], \hat{P}=\left[\begin{array}{cc}
\frac{I}{a} & 0 \\
0 & I
\end{array}\right]\left[\begin{array}{cc}
\sqrt{2} a I & I \\
I & \frac{\sqrt{2}}{a} I
\end{array}\right]\left[\begin{array}{cc}
\frac{I}{a} & 0 \\
0 & I
\end{array}\right]=\frac{1}{a}\left[\begin{array}{cc}
\sqrt{2} I & I \\
I & \sqrt{2} I
\end{array}\right], \\
& \hat{P}^{-1}=a\left[\begin{array}{cc}
\sqrt{2} I & -I \\
-I & \sqrt{2} I
\end{array}\right], \quad-\left(\hat{A}_{2 i}{ }^{T} \hat{P}+\hat{P} \hat{A}_{2 i}\right) \hat{P}^{-1}=\left[\begin{array}{cc}
A_{2 i} & -\sqrt{2} A_{2 i} \\
\sqrt{2} A_{2 i} & -2 A_{2 i}-A_{2 i}^{T}
\end{array}\right] .
\end{aligned}
$$

From (69), (81) and (82) the relation (74) easily follows.

Relations (75) easily follow from the third of (59), from (70), from the third of (72) and by considering (78).

Remark 10. It is easy to note that the values of $c$ and $c_{p}$ provided by (75) are the same if, instead of $y$ and $\dot{y}$, their components $y_{i}$ e $\dot{y}_{i}$ are considered.

Now the main result can be stated. It allows determining the control law which guarantees prefixed majorant values of the time constant $\tau=\frac{1}{\alpha_{2}\left(\rho_{2}-\rho_{1}\right)}$ related to $\varphi(t)$ and of the time constant $\tau_{l}=-\frac{1}{\alpha_{1}}$ of the linearized majorant system and prefixed majorant values of the "steady-state" ones of $\left|y_{i}\right|$ e $\left|\dot{y}_{i}\right|$.

Theorem 18. If system (56) is controlled by using the control law

$$
u=-K\left(a^{2} y+\sqrt{2} a \dot{y}\right)-u_{c}
$$


with $K, a, u_{c}$ such that

where

$$
\lambda_{\min }\left(K^{T} F_{1}^{T}+F_{1} K\right) \geq 2, \quad a \geq 2.463 \sqrt[5]{\alpha_{2}^{2} d^{2}},
$$

$$
\begin{gathered}
d=\max _{t \in R, x \in C P, \rho, p \in \rho \rho}\left\|f-F_{1} u_{c}\right\|, \quad \alpha_{2}=-\min _{x \in \mathcal{C}, 1, p \in \rho} \frac{\lambda_{\min }\left(\sum_{i=1}^{m}\left[\begin{array}{cc}
A_{2 i} & -\sqrt{2} A_{2 i} \\
\sqrt{2} A_{2 i} & -2 A_{2 i}-A_{2 i}^{T}
\end{array}\right] x_{m+i-1}\right)}{2}>0, \\
P=\left[\begin{array}{cc}
\sqrt{2} a I & I \\
I & \frac{\sqrt{2}}{a} I
\end{array}\right],
\end{gathered}
$$

said $\rho_{1}, \rho_{2}$ the roots of the equation

$$
\alpha_{2} \rho^{2}-\frac{a}{\sqrt{2}} \rho+\frac{\sqrt[4]{2}}{\sqrt{a}} d=0
$$

$\forall x_{0}:\left\|x_{0}\right\|_{P}=\sqrt{x_{0}^{T} P x_{0}}<\rho_{2}$ it is:

$$
\begin{aligned}
& \left|y_{i}(t)\right| \leq \frac{\sqrt[4]{2}}{\sqrt{a}} \rho(t), \quad\left|\dot{y}_{i}(t)\right| \leq \sqrt[4]{2} \sqrt{a} \rho(t), \quad \rho(t)=\frac{\rho_{1}-\rho_{2} \varphi(t)}{1-\varphi(t)} \\
& \text { where } \varphi(t)=\frac{\rho_{0}-\rho_{1}}{\rho_{0}-\rho_{2}} e^{-t / \tau}, \tau=\frac{1}{\alpha_{2}\left(\rho_{2}-\rho_{1}\right)}
\end{aligned}
$$

with time constant $\tau_{l}=\frac{\sqrt{2}}{a}$; moreover, for $a$ big enough such that $\rho_{1} \ll \rho_{2}$, it is:

$$
\lim _{t \rightarrow \infty}\left|y_{i}(t)\right| \leq \frac{\sqrt[4]{2}}{\sqrt{a}} \rho_{1} \cong \frac{2}{a^{2}} d, \quad \lim _{t \rightarrow \infty}\left|\dot{y}_{i}(t)\right| \leq \sqrt[4]{2} \sqrt{a} \rho_{1} \cong \frac{2}{a} d, \quad \tau \cong \frac{\sqrt{2}}{a}=\tau_{l} .
$$

Proof. The proof of (87) follows from Theorems 13, 16 and 17. The proof of (88) derives from the fact that if $\rho_{1} \ll \rho_{2}$ it is $\rho_{1} \cong \frac{\sqrt[4]{2}}{\sqrt{a}} / \frac{a}{\sqrt{2}}, \rho_{2}-\rho_{1} \cong \frac{a}{\alpha_{2} \sqrt{2}}$.

Remark 11. As regards the determination of $K$ in order to satisfy the first of (84), the computation of $u_{c}$ to decrease $d$ and regarding the computation of $\alpha_{2}$ and $d$, for limitation of pages, it has to be noted at least that for the mechanical systems, being $F_{1}=B^{-1}$, taking into account that the inertia matrix $B$ is symmetric and $p . d . \forall p \in \wp$ and $\forall y=q \in R^{m}$, under the hypothesis that $T=I$ it can be chosen $K=k I$, with $k \geq \lambda_{\max }(B)$. Moreover it can be posed $u_{c}=g(t, y, \hat{p})$, with $\hat{p}$ nominal value of the parameters. Finally the calculation of $\lambda_{\max }(B), \alpha_{2}$ and $d$ can be facilitated by suitably using Theorem 15 .

Remark 12. The stated theorems can be used for determining simple and robust control laws of the PD type, with a possible compensation action, in order to force system (56) to track a 
generic preassigned limited in "acceleration" trajectory, with preassigned increases of the maximum "position and/or velocity" errors and preassigned increases of the time constants characterizing the convergence of the error.

\section{Conclusion}

In this chapter it is has been considered one of the main and most realistic control problem not suitable solved in literature (to design robust control laws to force an uncertain parametric system subject to disturbances to track generic references but regular enough with a maximum prefixed error starting from a prefixed instant time).

This problem is satisfactorily solved for SISO processes, without zeros, with measurable state and with parametric uncertainties by using theorems and algorithms deriving from some proprierties of the most common filters, from Kharitonov's theorem and from the theory of the externally positive systems.

The considered problem has been solved also for a class of uncertain pseudo-quadratic systems, including articulated mechanical ones, but for limitation of pages only the two fundamental results have been reported. They allow to calculate, by using efficient algorithms, the parameters characterizing the performances of the control system as a function of the design parameters of the control law.

\section{References}

Butterworth, S. (1930). On the Theory of Filter Amplifiers. Experimental Wireless and the Wireless Engineering, no. 7, pp. 536-541

Porter, B. and Power, H.M. (1970). Controllability of Multivariable Systems Incorporating Integral Feedback. Electron. Lett., no. 6, pp. 689-690

Seraj, H. and Tarokh, M. (1977). Design of Proportional-Plus-Derivative Output Feedback for Pole Assignement. Proc. IEE, vol. 124, no. 8, pp. 729-732

Ambrosino, G., Celentano, G. and Garofalo, F. (1985). Robust Model Tracking Control for a Class of Nonlinear Plants. IEEE Trans. Autom. Control, vol. 30, no. 3, pp. 275-279

Dorato P. (Editor) (1987). Robust control, IEEE Press

Jayasuriya, S. and Hwang, C.N. (1988). Tracking Controllers for Robot Manipulators: a High Gain Perspective. ASME J. of Dynamic Systems, Measurement, and Control, vol. 110, pp. 39-45

Nijmeijer, H. and Van der Schaft, A. J. (1990). Nonlinear Dynamical Control Systems, SpringerVerlag

Slotine, J. J. E. and Li, W. (1991). Applied nonlinear control, Prentice-Hall

Tao, G. (1992). On Robust Adaptive Control of Robot Manipulators. Automatica, vol. 28, no. 4, pp. 803-807.

Colbaugh, R., Glass, K. and Seraji, H. (1993). A New Approach to Adaptive Manipulator Control. Proc. IEEE Intern. Conf. on Robotics and Automation, vol. 1, pp. 604-611, Atlanta

Abdallah, C. T., Dorato, P. and Cerone, V. (1995). Linear Quadratic Control, Englewood Cliffs, New Jersey, Prentice-Hall.

Freeman, R.A. and Kokotovic, P.V., (1995). Robust Integral Control for a Class of Uncertain Nonlinear systems. 34th IEEE Intern. Conf. on Decision E Control, pp. 2245-2250, New Orleans 
Arimoto, S. (1996). Control Theory of Nonlinear Mechanical Systems, Oxford Engineering Science Series

Sastry, S. (1999). Nonlinear Systems, Analysis, Stability and Control, Springer-Verlag

Paarmann, L.D. (2001). Design and Analysis of Analog Filters: A Signal Processing Perspective, Kluwer Academic Publishers, Springer

Celentano, L. (2005). A General and Efficient Robust Control Method for Uncertain Nonlinear Mechanical Systems. Proc. IEEE Conf. Decision and Control, Seville, Spain, pp. 659-665

Amato, F. (2006). Robust Control of Linear Systems Subject to Uncertain Time-Varying Parameters, Springer-Verlag

Siciliano, S. and Khatib, O. (Editors) (2009). Springer Handbook of Robotics, Springer

Bru, R. and Romero-Vivò, S. (2009). Positive Systems. Proc. 3rd Multidisciplinary Intern. Symposium on Positive Systems: Theory and Applications, Valencia, Spain 


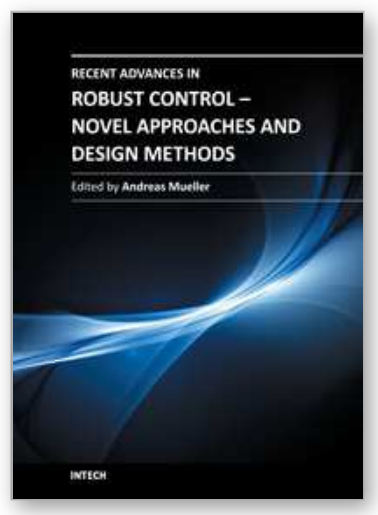

\author{
Recent Advances in Robust Control - Novel Approaches and \\ Design Methods \\ Edited by Dr. Andreas Mueller
}

ISBN 978-953-307-339-2

Hard cover, 462 pages

Publisher InTech

Published online 07, November, 2011

Published in print edition November, 2011

Robust control has been a topic of active research in the last three decades culminating in $\mathrm{H}$ 2/H_linfty and Imu design methods followed by research on parametric robustness, initially motivated by Kharitonov's theorem, the extension to non-linear time delay systems, and other more recent methods. The two volumes of Recent Advances in Robust Control give a selective overview of recent theoretical developments and present selected application examples. The volumes comprise 39 contributions covering various theoretical aspects as well as different application areas. The first volume covers selected problems in the theory of robust control and its application to robotic and electromechanical systems. The second volume is dedicated to special topics in robust control and problem specific solutions. Recent Advances in Robust Control will be a valuable reference for those interested in the recent theoretical advances and for researchers working in the broad field of robotics and mechatronics.

\title{
How to reference
}

In order to correctly reference this scholarly work, feel free to copy and paste the following:

Laura Celentano (2011). New Robust Tracking and Stabilization Methods for Significant Classes of Uncertain Linear and Nonlinear Systems, Recent Advances in Robust Control - Novel Approaches and Design Methods, Dr. Andreas Mueller (Ed.), ISBN: 978-953-307-339-2, InTech, Available from:

http://www.intechopen.com/books/recent-advances-in-robust-control-novel-approaches-and-designmethods/new-robust-tracking-and-stabilization-methods-for-significant-classes-of-uncertain-linear-and-nonlin

\section{INTECH}

open science | open minds

\section{InTech Europe}

University Campus STeP Ri

Slavka Krautzeka 83/A

51000 Rijeka, Croatia

Phone: +385 (51) 770447

Fax: +385 (51) 686166

www.intechopen.com

\section{InTech China}

Unit 405, Office Block, Hotel Equatorial Shanghai

No.65, Yan An Road (West), Shanghai, 200040, China

中国上海市延安西路65号上海国际贵都大饭店办公楼 405 单元

Phone: +86-21-62489820

Fax: $+86-21-62489821$ 
(C) 2011 The Author(s). Licensee IntechOpen. This is an open access article distributed under the terms of the Creative Commons Attribution 3.0 License, which permits unrestricted use, distribution, and reproduction in any medium, provided the original work is properly cited. 